\title{
Video Article \\ Isolation and Characterization of Microvesicles from Peripheral Blood
}

\author{
Kerstin Menck ${ }^{1}$, Annalen Bleckmann ${ }^{1}$, Matthias Schulz ${ }^{1}$, Lena Ries ${ }^{1}$, Claudia Binder \\ ${ }^{1}$ Department of Hematology/Medical Oncology, University Medical Center Göttingen
}

Correspondence to: Kerstin Menck at kerstin.menck@inserm.fr

URL: https://www.jove.com/video/55057

DOI: doi:10.3791/55057

Keywords: Cellular Biology, Issue 119, extracellular vesicles, microvesicles, blood, flow cytometry, biomarkers, liquid biopsy, cancer

Date Published: 1/6/2017

Citation: Menck, K., Bleckmann, A., Schulz, M., Ries, L., Binder, C. Isolation and Characterization of Microvesicles from Peripheral Blood. J. Vis. Exp. (119), e55057, doi:10.3791/55057 (2017).

\section{Abstract}

The release of extracellular vesicles (EVs) including small endosomal-derived exosomes (Exos, diameter $<100 \mathrm{~nm}$ ) and large plasma membrane-derived microvesicles (MVs, diameter $>100 \mathrm{~nm}$ ) is a fundamental cellular process that occurs in all living cells. These vesicles transport proteins, lipids and nucleic acids specific for their cell of origin and in vitro studies have highlighted their importance as mediators of intercellular communication. EVs have been successfully isolated from various body fluids and especially EVs in blood have been identified as promising biomarkers for cancer or infectious diseases. In order to allow the study of MV subpopulations in blood, we present a protocol for the standardized isolation and characterization of MVs from peripheral blood samples. MVs are pelleted from EDTA-anticoagulated plasma samples by differential centrifugation and typically possess a diameter of $100-600 \mathrm{~nm}$. Due to their larger size, they can easily be studied by flow cytometry, a technique that is routinely used in clinical diagnostics and available in most laboratories. Several examples for quality control assays of the isolated MVs will be given and markers that can be used for the discrimination of different MV subpopulations in blood will be presented.

\section{Video Link}

The video component of this article can be found at https://www.jove.com/video/55057/

\section{Introduction}

In the last years many in vitro studies have demonstrated that extracellular vesicles (EVs) play an important role in intercellular communication. Living cells constantly shed vesicles which differ in size, content and biogenesis. The best studied EVs are exosomes which originate from the endosomal system where they are stored as intraluminal vesicles in multivesicular bodies. Once the latter fuse with the plasma membrane, the contained vesicles are released as exosomes (Exos, diameter $30-100 \mathrm{~nm}^{1}$ ). A second population of EV which has gained increasing attention in the last years are large microvesicles (MVs, diameter $100-1,000 \mathrm{~nm}$ ) which bud off directly from the plasma membrane ${ }^{2}$.

Both types of vesicles are surrounded by a lipid bilayer and contain nucleic acids, e.g., DNA, mRNA or miRNA ${ }^{3-5}$, and a plethora of proteins which they can transfer to neighboring cells. While in general the protein composition of the vesicles reflects the state of the cell of origin, some proteins seem to be selectively targeted and enriched on EVs ${ }^{1}$. A major research interest is to characterize EVs from abnormal and diseased cells in order to define specific EV signatures which might allow the use of EVs as novel biomarkers. Especially in cancer, in which often the tumor itself is not easily accessible, liquid biopsies targeting tumor-specific EVs in blood might allow monitoring of therapy responses or help characterizing the primary tumor without the need for invasive procedures ${ }^{6}$.

Indeed, EVs have already been successfully isolated from various body fluids including urine ${ }^{7}, \mathrm{CSF}^{8}$, breast milk ${ }^{9}$ or blood ${ }^{10}$. Several studies identified changes in EV counts and composition in different human diseases. For example, in sepsis patients the number of pro-coagulant MVs is significantly increased compared to healthy individuals ${ }^{11}$. Also in patients with severe cerebral malaria an increase in total MVs in blood can be observed and counts of platelet-derived MVs correlate with coma depth and thrombocytopenia ${ }^{12}$. Other studies report elevated numbers of endothelium-derived vesicles in patients with systemic lupus erythematosus or heart failure and in the case of the latter, this correlates with a higher probability of cardiovascular events ${ }^{13,14}$.

Especially in cancer, EVs in blood are currently discussed as novel biomarkers with diagnostic and prognostic value. Levels of MVs expressing tumor-associated proteins such as MUC1, EGFR or FAK seem to be elevated in the blood of breast cancer patients ${ }^{15,16}$. Also for Exos, recent studies have shown that blood-derived Exos carrying tumor-specific antigens such as Glypican-1 for pancreatic cancer or Del-1 for breast cancer allow early disease detection with high specificity and sensitivity ${ }^{17,18}$. Additionally, serum-derived tumor Exos may contain DNA which can be used for detection of mutations such as KRAS and p53 which suggests their use for therapy prediction ${ }^{19}$. Recent advances have shown that analysis of Exos in blood of glioblastoma patients using a specific microfluidic chip allows monitoring of therapy ${ }^{20}$. Taken together, these findings imply that analysis of disease-specific subpopulations of vesicles gives valuable information about diagnosis, prognosis as well as therapeutic options and success.

However, isolation and analysis of Exos from blood is time-consuming, requires special lab equipment and therefore is not yet suited for routine clinical diagnostics. In contrast, MVs can be isolated much faster and, due to their larger size, can be easily analyzed by flow cytometry without 
the need to couple them to latex beads as it is necessary for Exos ${ }^{18,21}$. Thus, here we present a protocol that can be used for the standardized isolation of MVs from blood samples and the subsequent characterization of MV subpopulations by flow cytometry. This protocol will allow the further study and in depth characterization of MV profiles in large patient groups which will be required in order to use MVs for everyday clinical diagnostics.

\section{Protocol}

All experiments including human subjects have been approved by the local ethics committee (approval no. 3/2/14). For the choice of patients it should be noted that several factors such as age, sex, current therapy regimens and many more may influence MV composition in blood and therefore should be taken into consideration prior to sample collection ${ }^{22,23}$.

\section{Preparation of Plasma Samples}

1. Draw 1 - 2 tubes of blood per donor through a 21-gauge butterfly needle into a vacutainer blood collection tube containing EDTA (1.6 mg/mL blood). Make sure to invert the tube(s) several times to guarantee efficient blood anticoagulation.

NOTE: The recommended volume of blood for subsequent flow cytometry and Western Blot analysis is 5 - $15 \mathrm{~mL}$. In order to prevent MV degradation and loss, blood samples should be handled $<30 \mathrm{~min}$ after blood withdrawal.

2. Prepare plasma samples by centrifuging the samples for $15 \mathrm{~min}$ at $1,200 \mathrm{xg}$ at room temperature (RT).

3. Apply a valve filter in order to help the separation of plasma (= upper layer) from remaining blood cells (= lower layer).

4. Transfer the plasma into a $15 \mathrm{~mL}$ tube.

5. Centrifuge for $15 \mathrm{~min}$ at $1,500 \mathrm{xg}, \mathrm{RT}$ to pellet larger cell debris and remove remaining platelets.

6. Transfer the supernatant into a $15 \mathrm{~mL}$ tube and directly proceed with $\mathrm{MV}$ isolation or store samples for up to 6 months at $-20^{\circ} \mathrm{C}$ NOTE: The presented protocol can also be used to isolate MVs (and Exos) from cell culture supernatants. In order to do so, cultivate cells at $60-80 \%$ confluency for $24-48 \mathrm{~h}$ in culture medium supplemented with vesicle-depleted FCS, and then collect the supernatant. Centrifuge for $5 \mathrm{~min}$ at $750 \mathrm{x} \mathrm{g}, 4^{\circ} \mathrm{C}$ to deplete residual floating cells, fill the supernatant into a new $15 \mathrm{~mL}$ tube and centrifuge again for 5 min at $1,500 \mathrm{x}$ $\mathrm{g}, 4{ }^{\circ} \mathrm{C}$ to pellet larger cell debris. This supernatant can then be used for the isolation of MVs as described in Step $2.1-2.16$

\section{Isolation of MVs}

1. Transfer the plasma sample in a suitable centrifugation tube. If necessary, fill up tube with PBS in order to dilute the sample and prevent collapse of thin-walled tubes during the centrifugation procedure.

2. Centrifuge for $35 \mathrm{~min}$ at $14,000 \times \mathrm{g}, 4^{\circ} \mathrm{C}$.

3. Decant supernatant, keep tubes turned upside-down and put on a paper towel. Wait $3-5$ min until all remaining supernatant has been soaked into the towel and thereby removed from the sample.

4. Resuspend the MV pellet in $1,000 \mu \mathrm{L}$ PBS, transfer to a $1.5 \mathrm{~mL}$ tube and centrifuge for $35 \mathrm{~min}$ at $14,000 \times \mathrm{g}, 4^{\circ} \mathrm{C}$ in a tabletop centrifuge.

5. Aspirate supernatant.

6. Resuspend the MV pellet in $50-500 \mu \mathrm{L}$ PBS, depending on the size of the pellet. Alternatively, lyse MVs directly, e.g., in RIPA buffer (150 $\mathrm{mM} \mathrm{NaCl} / 0.1 \% \mathrm{SDS} / 0.5 \%$ Na-deoxycholate/ $1 \%$ Triton X-100/ $50 \mathrm{mM}$ Tris, $\mathrm{pH} 7.2$ ) for subsequent Western Blot analysis. Store MVs at -20 ${ }^{\circ} \mathrm{C}$. They will remain stable for several months, but avoid repeated freeze-thaw-cycles.

7. Optional: Determine the MV protein concentration with a protein assay (e.g., Bradford or Lowry method) in order to assess MV yields or dose MVs for subsequent experiments.

8. If additional Exos are to be isolated from the plasma samples, decant the supernatant from step 2.3 into an ultracentrifugation tube and centrifuge for $2 \mathrm{~h}$ at $110,000 \times \mathrm{g}, 4^{\circ} \mathrm{C}$. Decant the supernatant as described in step 2.3, resuspend Exo pellet in 1,000 $\mu \mathrm{L}$ PBS and transfer into small $(1.5 \mathrm{~mL})$ ultracentrifugation tubes.

1. Ultracentrifuge for $2 \mathrm{~h}$ at $110,000 \times \mathrm{g}, 4{ }^{\circ} \mathrm{C}$, aspirate supernatant and resuspend Exo pellet in $50-75 \mu \mathrm{L}$ PBS or RIPA buffer.

\section{Characterization of MVs by Flow Cytometry}

1. Transfer $15 \mu \mathrm{L}$ PBS $+1 \%$ vesicle-depleted fetal calf serum (FCS) in a flow cytometry tube. NOTE: Vesicle-depleted FCS is prepared by centrifuging heat-inactivated ( 30 min at $56{ }^{\circ} \mathrm{C}$ ) FCS for $18 \mathrm{~h}$ at $110,000 \times \mathrm{g}$ and filtrating the supernatant through a $0.2 \mu \mathrm{m}$ filter as described previously ${ }^{24}$.

2. Add $5 \mu \mathrm{g}$ (in case of low yields $3 \mu \mathrm{g}$ are also applicable) of MVs in PBS

3. Incubate samples for $30 \mathrm{~min}$ at RT in order to block unspecific binding sites at the MV surface and thereby reduce background staining.

4. Add a fluorescently labeled antibody against the protein-of-interest. Titrate the amount of antibody used for the staining prior to use in order to determine the optimal concentration and guarantee a low signal-to-noise ration. Make sure to also include one tube with unstained MVs as negative control and one tube of MVs stained with the matching isotype control antibody at the same concentration (e.g., if $1 \mu \mathrm{g}$ of antibody is used, also use $1 \mu \mathrm{g}$ of the isotype control antibody) to quantify background staining.

NOTE: It is also possible to perform multicolor flow cytometry by adding multiple antibodies coupled to different fluorochromes.

5. Incubate for 20 min at RT in the dark.

6. Add $250 \mu \mathrm{L}$ PBS and proceed with measurement of the sample using a flow cytometer.

1. In case that samples cannot be measured immediately, add $150 \mu \mathrm{L}$ PBS and $50 \mu \mathrm{L} 4 \%$ paraformaldehyde (PFA) to fix samples and store at $4{ }^{\circ} \mathrm{C}$. CAUTION: PFA is toxic. Use gloves and suitable personal protective equipment.

7. Reduce the threshold of the flow cytometer to the lowest value possible and search for the MV population using a forward scatter (FSC) versus side scatter (SSC) plot in logarithmic scale. Gate on the MV population and evaluate the fluorescent signal in a corresponding histogram. 


\section{Characterization of MVs by Western Blotting}

1. Resuspend the MV pellet directly in a suitable lysis buffer (e.g., RIPA buffer).

1. In case the MV pellet has already been resuspended in PBS, dilute it at least 1:1 in a suitable lysis buffer (e.g., RIPA buffer).

2. Determine the protein concentration of the MV sample, e.g., by Lowry assay.

3. Prepare $10-20 \mu \mathrm{g}$ of MVs in $22.5 \mu \mathrm{L}$ RIPA buffer. Then add $7.5 \mu \mathrm{L} 4 \mathrm{x}$ Laemmli loading buffer and heat for $5 \mathrm{~min}$ at $95^{\circ} \mathrm{C}$.

4. Load samples on a polyacrylamide gel and perform electrophoresis and immunoblotting according to standard protocols.

5. After transferring the proteins onto the membrane, perform a Ponceau staining as a loading control according to standard protocols.

6. Destain membrane in TBST for 5 min at RT.

7. Block membrane for $30 \mathrm{~min}$ up to $1 \mathrm{~h}$ at RT in $5 \% \mathrm{BSA}$ in TBST.

8. Incubate membrane with the primary antibody at $4{ }^{\circ} \mathrm{C}$ overnight or for $2 \mathrm{~h}$ at RT.

9. Wash the membrane with TBST $3 \times 5$ min.

10. Incubate membrane with the HRP-coupled secondary antibody at a dilution of $1: 10,000$ in $5 \%$ BSA. Note: In case of high background signals, use milk powder instead of BSA.

11. Wash the membrane with TBST $3 \times 5$ min.

12. Develop membrane with an ECL detection reagent and detect signals on chemiluminescence films or a chemiluminescence imaging system. NOTE: In order to discriminate MVs from Exos, proteins like Tubulin, actinin-4 or mitofilin can be used which should mainly be present on MVs ${ }^{16,25}$. Pay attention that most tetraspanin antibodies (e.g., CD9, CD81), used as markers for Exos, do not work under reducing conditions and should therefore be prepared in non-reducing loading buffer followed by heating for $10 \mathrm{~min}$ at $70^{\circ} \mathrm{C}$.

\section{Representative Results}

In order to quantify the yield of MVs that can be isolated following the described protocol, we calculated the amount of MVs isolated from blood samples of 10 donors. The MV yield, which was assessed in a Lowry protein assay, ranged from 10 up to $30 \mu \mathrm{g}$ with a mean of $19.2 \mu \mathrm{g}$ MVs per $\mathrm{mL}$ blood (Table 1). The particle concentration determined by nanoparticle tracking analysis (NTA) ranged from $1.66 \times 10^{9}$ to $2.36 \times 10^{10}$ with a mean of $5.9 \times 10^{9}$ particles per $\mathrm{mL}$ plasma sample (Table 2). Further characterization of the MVs by transmission electron microscopy revealed a population of vesicles with a diameter $>100 \mathrm{~nm}$ that were surrounded by a lipid bilayer and did not contain any cell organelles (Figure 1A). NTA confirmed that the size of the isolated MVs ranged from 100 up to $600 \mathrm{~nm}$ (Figure 1B) and the mean MV size was $201 \mathrm{~nm}$ (Figure 1C). Staining for typical MV and Exo markers by Western Blotting demonstrated that the isolated MVs were positive for Tubulin and only showed a slight expression of CD9 and CD81, while Exos were negative for Tubulin and enriched in CD9 and CD81 (Figure 2).

Analysis of the isolated MVs by flow cytometry (Figure 3A) revealed a defined vesicle population that could be gated using the same parameters normally used for MVs isolated from cell culture supernatants and that was clearly different from the background signal obtained by the measurement of PBS $+1 \%$ vesicles-depleted FCS without addition of MVs (Figure 3B). In order to analyze the different MV populations present in blood, MVs were stained with established markers for the different blood cell populations, e.g., CD62P for platelet-derived MVs, CD45 for leukocyte-derived MVs, CD235a for red blood cell-derived MVs and CD62E for endothelial cell-derived MVs (Figure 4). This characterization showed that the percentage of MV subpopulations differed among the investigated donor blood samples, while the majority of MVs seemed to be shed by platelets in all samples.
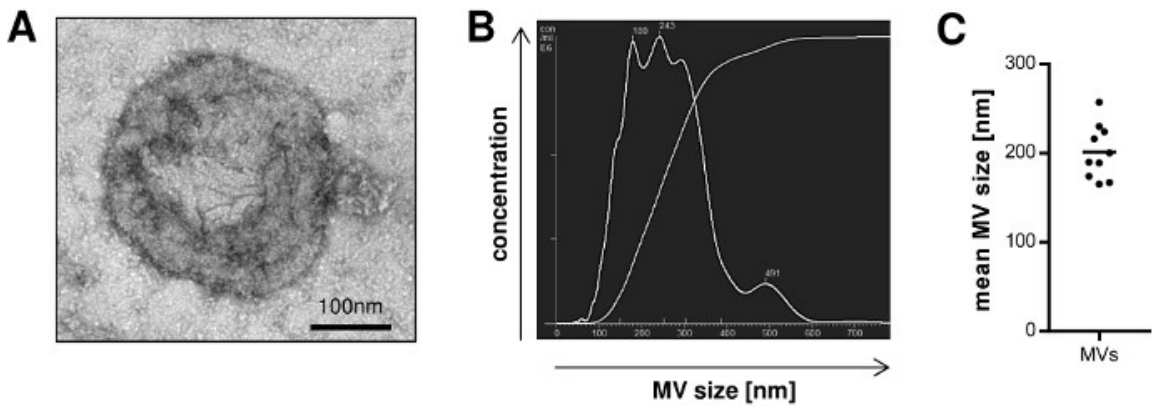

Figure 1: Size Distribution of MVs Isolated from Peripheral Blood. A, Isolated MVs were visualized by transmission electron microscopy. $\boldsymbol{B}$, Representative nanoparticle tracking analysis (NTA) of MVs illustrating the size distribution of the vesicles. $\boldsymbol{C}$, The mean MV size from 10 independent preparations was measured by NTA (mean). Please click here to view a larger version of this figure. 


\section{donor 1 donor 2}

\section{Exo MV Exo}

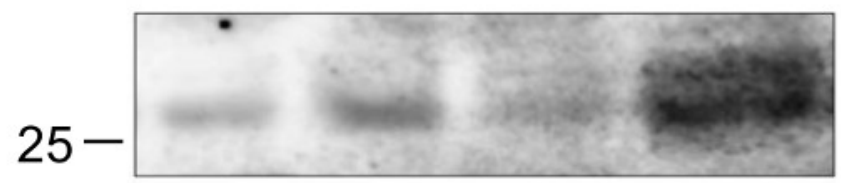

\section{CD81}

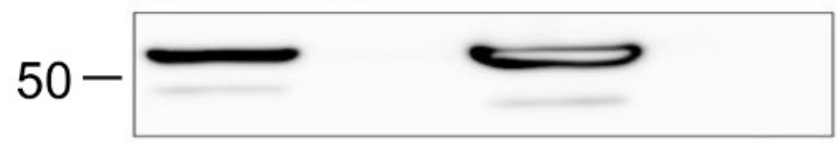

\section{Tubulin}

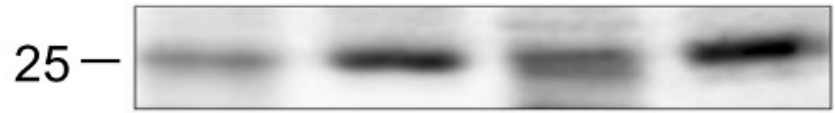

CD9

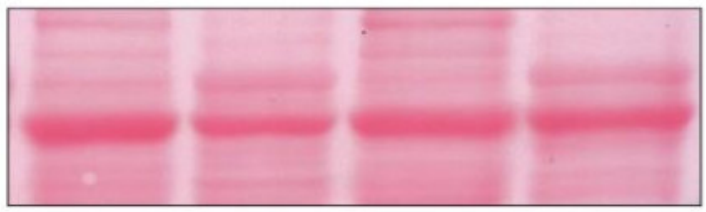

\section{Ponceau}

Figure 2: Characterization of Isolated MVs by Western Blotting. Differential protein expression on the isolated MVs and Exos from two donors was visualized by Western Blotting. Please click here to view a larger version of this figure.
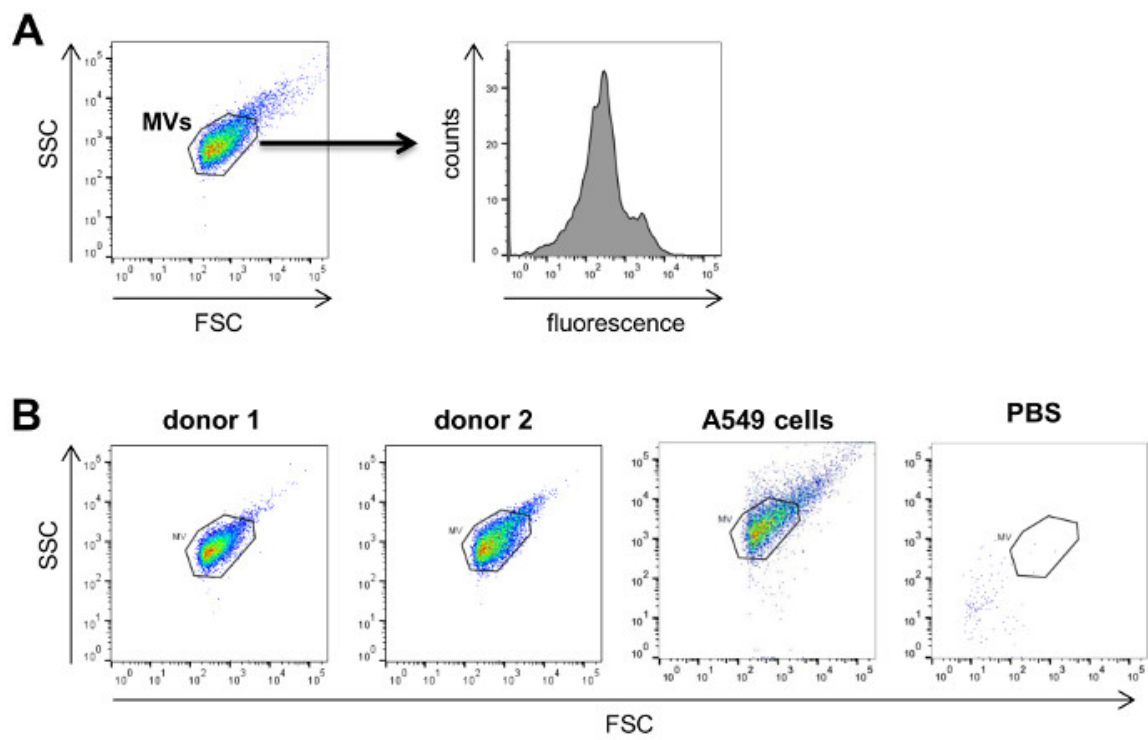

Figure 3: Analysis of MVs by Flow Cytometry. A, MVs are first visualized on forward (FSC) versus sidescatter (SSC) plots to gate on the respective MV population. Subsequently, these MVs are characterized for the antigen of interest by using fluorescently labeled antibodies directed against the antigen. $\boldsymbol{B}$, Typical FSC versus SSC plots for MVs isolated from the plasma of two donors. As comparison, a typical plot for tumor cell-derived MVs from A549 lung cancer cells isolated from cell culture supernatant as well as a negative control using only PBS $+1 \%$ vesicle-depleted FCS without MVs are shown on the right. Please click here to view a larger version of this figure. 

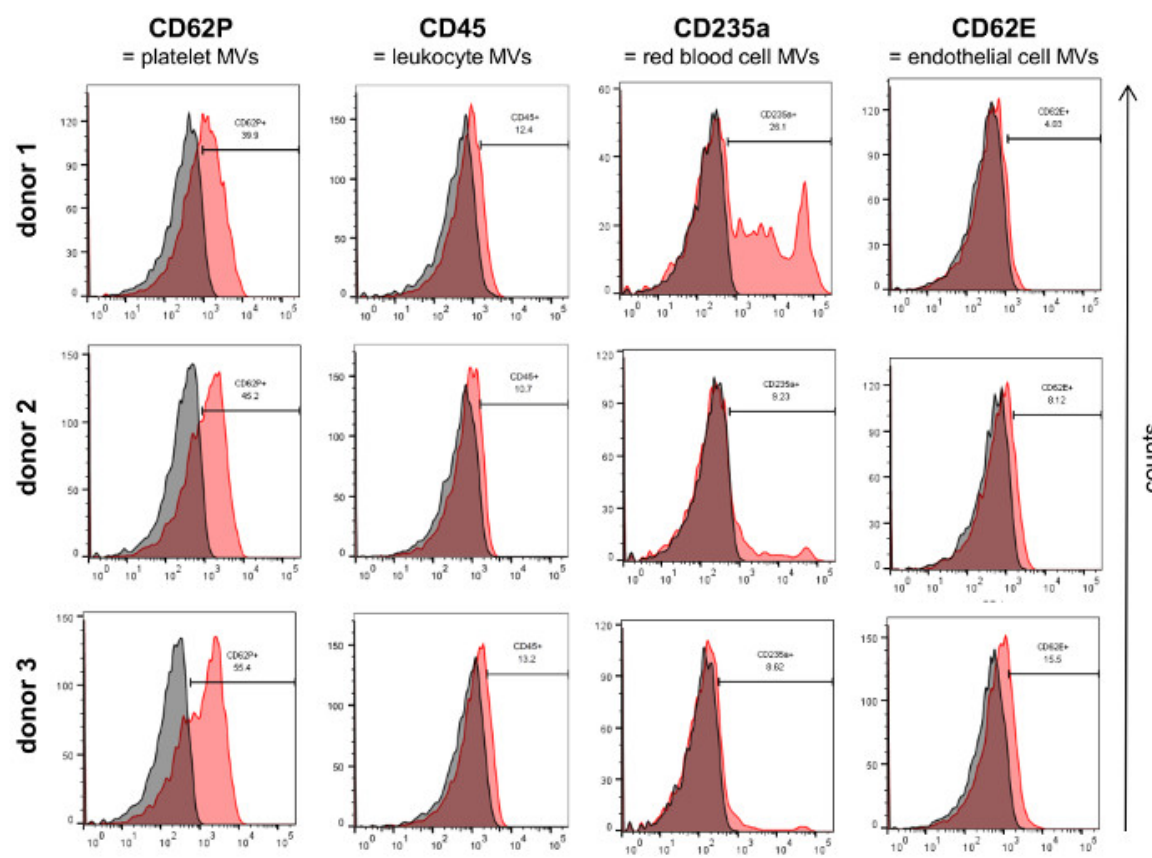

Figure 4: Characterization of Isolated MVs by Flow Cytometry. MVs from three donors were characterized for the expression of established blood cell markers (red) by flow cytometry. The respective isotype controls are shown in grey. Please click here to view a larger version of this figure.

\begin{tabular}{|l|l|}
\hline Sample & MVs/mL blood [ $\boldsymbol{\mu g}]$ \\
\hline$\# 1$ & 29.4 \\
\hline$\# 2$ & 10.3 \\
\hline$\# 3$ & 15.2 \\
\hline$\# 4$ & 31.1 \\
\hline$\# 5$ & 18.8 \\
\hline$\# 6$ & 22.7 \\
\hline$\# 7$ & 19.1 \\
\hline$\# 8$ & 18.7 \\
\hline$\# 9$ & 15.0 \\
\hline$\# 10$ & 11.9 \\
\hline
\end{tabular}

Table 1: MV Protein Yield from Peripheral Blood Samples. Shown is the amount of MVs per mL peripheral blood that was drawn from 10 donors. MV yields were quantified by Lowry assay.

\begin{tabular}{|l|l|}
\hline Sample & MVs/mL plasma [particle count] \\
\hline$\# 1$ & $6.42 \mathrm{E}+09$ \\
\hline$\# 2$ & $2.36 \mathrm{E}+10$ \\
\hline$\# 3$ & $1.88 \mathrm{E}+09$ \\
\hline$\# 4$ & $6.51 \mathrm{E}+09$ \\
\hline$\# 5$ & $3.48 \mathrm{E}+09$ \\
\hline$\# 6$ & $4.57 \mathrm{E}+09$ \\
\hline$\# 7$ & $2.09 \mathrm{E}+09$ \\
\hline$\# 8$ & $1.66 \mathrm{E}+09$ \\
\hline$\# 9$ & $2.54 \mathrm{E}+09$ \\
\hline$\# 10$ & $6.20 \mathrm{E}+09$ \\
\hline
\end{tabular}

Table 2: MV Particle Yield from Peripheral Blood Samples. MV were isolated from plasma samples of 10 donors and particle counts were determined by nanoparticle tracking analysis. Shown is the mean value from three independent measurements. 


\section{Discussion}

Recent studies on EVs in blood have demonstrated that EV composition and counts change during the cause of several diseases. Therefore, the analysis and further characterization of these EVs are of high interest to further assess their potential use as disease biomarkers for diagnosis and prognosis or to evaluate therapy responses. The protocol we present here allows the isolation of vesicles with a diameter of up to $600 \mathrm{~nm}$ which do not contain any cell organelles. These observations are in line with the current definition of MVs and exclude the presence of apoptotic bodies $^{2}$. Using Western Blotting, we were able to demonstrate that the isolated MVs show a high expression of Tubulin, while the tetraspanins CD9 and CD81 that are often used as Exo markers were only slightly expressed. This confirms that MVs differ from Exos and fits to recent indepth characterization and comparison of both EV populations by proteomics ${ }^{25}$

During acquisition of blood samples, it is critical to keep the time between venipuncture and plasma preparation as short as possible in order to prevent MV degradation. Moreover, prolonged storage of blood samples could lead to the activation of blood cells causing enhanced MV shedding and ultimately apoptosis which results in the release of apoptotic bodies. Another important consideration for MV isolation is to prevent contaminations of MV preparations with plasma proteins or smaller Exos. Therefore, it is critical to remove as much of the supernatant as possible after spinning down the MVs at $14,000 \times \mathrm{g}$. Since the pellet is normally visible and tightly attached to the wall of the tube, the supernatant can be easily removed with a pipette tip. In contrast to Exos which tend to form aggregates during preparation by high speed ultracentrifugation and are often hard to resuspend, this problem does not occur with MVs.

Our study shows that it is possible to characterize MV subpopulations present in blood by flow cytometry. Although the detection limit of most flow cytometers is around $200-300 \mathrm{~nm}$, MVs were reproducibly measured in donor as well as cell culture samples with the same parameters of analysis and gates that clearly allowed their distinction from background signals. It is important to verify prior to the measurements that the PBS used for the analyses does not contain any contaminating particles that could cause a high background during flow cytometry (Figure 3). Although some smaller MVs might not be captured in a flow cytometric approach, we detected MVs from all major blood cell populations (e.g., platelets, red blood cells, leukocytes, endothelial cells). In our analyses we used standard markers for the different blood cells that have been previously found on $\mathrm{MVs}{ }^{26-29}$. It should be noted that in order to obtain the best possible results by flow cytometry, the amount and concentration of all antibodies should be titrated on a MV sample expressing the antigen of interest. If specific MV subpopulations in blood shall be identified with higher specificity, it is possible to perform double staining against two different antigens present on the respective MVs and only consider all double positive MVs for subsequent analyses ${ }^{23}$. Currently, there are efforts to define a standard range of MV subpopulations in blood of healthy individuals ${ }^{23,30}$. These studies have already shown that platelet-derived MVs constitute the largest population of MVs in blood which is in correspondence with our observations.

One advantage of flow cytometry to characterize MV samples is that this method is already well established for diagnostic purposes in most clinical centers which would allow the possible use of MVs as biomarkers in everyday clinical diagnostics. Previous studies on EVs in blood which have mostly focused on smaller Exos, rely on either specific sorting procedures to selectively analyze the desired Exo target population 31,32 or require a time-consuming ( 2 days) isolation process with coupling of Exos to Latex beads prior to analysis ${ }^{18}$. Our own unpublished observations suggest that the flow cytometric analysis of MVs from whole blood preparations even allows the detection of MVs such as tumorderived MVs without any such selection processes.

Taken together, the protocol presented here allows the fast isolation of MVs from peripheral blood samples with standard lab equipment and their subsequent characterization using flow cytometry and Western Blotting. The whole process can be performed in around $2 \mathrm{~h}$ which will facilitate future studies on MV profiles in patients' blood that are required to assess the potential of MVs as disease biomarkers.

\section{Disclosures}

The authors have nothing to disclose.

\section{Acknowledgements}

The authors acknowledge Meike Schaffrinski for excellent technical assistance. We would like to thank the following people for their help in the collection of peripheral blood samples (all from University Medical Center Göttingen): Henrietta Vida (Dept. of Transfusion Medicine), Kia Homayounfar, Lena-Christin Conradi (Dept. of General, Visceral and Pediatric Surgery), Leila Siam, Bawarjan Schatlo (Dept. of Neurosurgery), Hendrik A. Wolff, Martin Canis (Dept. of Otorhinolaryngology, Head and Neck Surgery) as well as all employees of the interdisciplinary short-term oncology. We acknowledge Dirk Wenzel (Max Planck Institute for Biophysical Chemistry, Göttingen) for his help with the electron microscopy of MVs.

The study was funded by the German Ministry of Education and Research (BMBF) project MetastaSys (grant no. 0316173) as well as the German Cancer Aid (grant no. 109615).

\section{References}

1. Raposo, G., \& Stoorvogel, W. Extracellular vesicles: Exosomes, microvesicles, and friends. J Cell Biol. 200 (4), $373-383$ (2013).

2. Yáñez-Mó, M., et al. Biological properties of extracellular vesicles and their physiological functions. J Extracell Vesicles. 4 (0) (2015).

3. Guescini, M., Genedani, S., Stocchi, V., \& Agnati, L. F. Astrocytes and Glioblastoma cells release exosomes carrying mtDNA. J Neural Transm (Vienna). 117 (1), 1-4 (2010).

4. Balaj, L., et al. Tumour microvesicles contain retrotransposon elements and amplified oncogene sequences. Nat Commun 2, 180 (2011). 
5. Lee, T. H., et al. Oncogenic ras-driven cancer cell vesiculation leads to emission of double-stranded DNA capable of interacting with target cells. Biochem Biophys Res Commun 451 (2), 295-301 (2014).

6. Chi, K. R. The tumour trail left in blood. Nature. 532 (7598), 269-271 (2016).

7. Pisitkun, T., Shen, R.-F., \& Knepper, M. A. Identification and proteomic profiling of exosomes in human urine. Proc Natl Acad Sci USA. 101 (36), 13368-13373 (2004).

8. Harrington, M. G., et al. The morphology and biochemistry of nanostructures provide evidence for synthesis and signaling functions in human cerebrospinal fluid. Cerebrospinal Fluid Res 6, 10 (2009).

9. Admyre, C., et al. Exosomes with Immune Modulatory Features Are Present in Human Breast Milk. J Immunol 179 (3), 1969-1978 (2007).

10. Caby, M.-P., Lankar, D., Vincendeau-Scherrer, C., Raposo, G., \& Bonnerot, C. Exosomal-like vesicles are present in human blood plasma. Int Immunol. 17 (7), 879-887 (2005).

11. Nieuwland, R., et al. Cellular origin and procoagulant properties of microparticles in meningococcal sepsis. Blood 95 (3), $930-935$ (2000).

12. Mfonkeu, J. B. P., et al. Elevated Cell-Specific Microparticles Are a Biological Marker for Cerebral Dysfunctions in Human Severe Malaria. PLoS One 5 (10), e13415 (2010).

13. Parker, B., et al. Suppression of inflammation reduces endothelial microparticles in active systemic lupus erythematosus. Ann Rheum Dis 73 (6), 1144-1150 (2014).

14. Nozaki, T., et al. Prognostic value of endothelial microparticles in patients with heart failure. Eur J Heart Fail 12 (11), $1223-1228$ (2010).

15. Galindo-Hernandez, O., et al. Elevated concentration of microvesicles isolated from peripheral blood in breast cancer patients. Arch Med Res 44 (3), 208-214 (2013).

16. Menck, K., et al. Tumor-derived microvesicles mediate human breast cancer invasion through differentially glycosylated EMMPRIN. J Mol Cell Biol 7 (2), 143-153 (2015).

17. Moon, P.-G., et al. Identification of Developmental Endothelial Locus-1 on Circulating Extracellular Vesicles as a Novel Biomarker for Early Breast Cancer Detection. Clin Cancer Res 22 (7), 1757-1766 (2016).

18. Melo, S. A., et al. Glypican-1 identifies cancer exosomes and detects early pancreatic cancer. Nature $\mathbf{5 2 3}$ (7559), 177-182 (2015).

19. Kahlert, C., et al. Identification of Double-stranded Genomic DNA Spanning All Chromosomes with Mutated KRAS and p53 DNA in the Serum Exosomes of Patients with Pancreatic Cancer. J Biol Chem 289 (7), 3869-3875 (2014).

20. Shao, H., et al. Protein typing of circulating microvesicles allows real-time monitoring of glioblastoma therapy. Nat Med 18 (12), $1835-1840$ (2012).

21. Eldh, M., \& Lötvall, J. Isolation and Characterization of RNA-Containing Exosomes. J Vis Exp. (59) (2012).

22. Witwer, K. W., et al. Standardization of sample collection, isolation and analysis methods in extracellular vesicle research. $J$ Extracell Vesicles 2 (0) (2013).

23. Gustafson, C. M., Shepherd, A. J., Miller, V. M., \& Jayachandran, M. Age- and sex-specific differences in blood-borne microvesicles from apparently healthy humans. Biol Sex Differ. 6 (2015).

24. Shelke, G. V., Lässer, C., Gho, Y. S., \& Lötvall, J. Importance of exosome depletion protocols to eliminate functional and RNA-containing extracellular vesicles from fetal bovine serum. J Extracell Vesicles. 3 (0) (2014).

25. Kowal, J., et al. Proteomic comparison defines novel markers to characterize heterogeneous populations of extracellular vesicle subtypes. Proc Natl Acad Sci USA 113 (8), E968-E977 (2016).

26. Dignat-George, F., \& Boulanger, C. M. The Many Faces of Endothelial Microparticles. Arterioscler Thromb Vasc Biol. 31 (1), $27-33$ (2011).

27. Esser, M. T., et al. Differential Incorporation of CD45, CD80 (B7-1), CD86 (B7-2), and Major Histocompatibility Complex Class I and II Molecules into Human Immunodeficiency Virus Type 1 Virions and Microvesicles: Implications for Viral Pathogenesis and Immune Regulation. J Virol 75 (13), 6173-6182 (2001).

28. Canellini, G., et al. Red blood cell microparticles and blood group antigens: an analysis by flow cytometry. Blood Transfus 10 (Suppl 2 ), s39s45 (2012).

29. Scholz, T., Temmler, U., Krause, S., Heptinstall, S., \& Lösche, W. Transfer of tissue factor from platelets to monocytes: role of platelet-derived microvesicles and CD62P. Thromb Haemost. 88 (6), 1033-1038 (2002).

30. Berckmans, R. J., et al. Cell-derived microparticles circulate in healthy humans and support low grade thrombin generation. Thromb Haemost 85 (4), 639-646 (2001).

31. Rupp, A.-K., et al. Loss of EpCAM expression in breast cancer derived serum exosomes: role of proteolytic cleavage. Gynecol Oncol 122 (2), 437-446 (2011).

32. Taylor, D. D., \& Gercel-Taylor, C. MicroRNA signatures of tumor-derived exosomes as diagnostic biomarkers of ovarian cancer. Gynecol Oncol. 110 (1), 13-21 (2008). 